\title{
Apuê: Uma Plataforma Web para Educação de Ciências Experimentais baseada em Tecnologias Livres
}

\author{
Apuê: A web platform for education of experimental sciences based on free technologies
}

\section{Gerson M. D. Almeida}

Universidade Tecnológica Federal do Paraná (UTFPR)

Av, Sete de setembro, 3165 - Curitiba - PR - Brasil

gersonmdda@gmail.com

Lucas S. Spolaore

Universidade Tecnológica Federal do Paraná (UTFPR)

Av, Sete de setembro, 3165 - Curitiba - PR - Brasil lucas.spolaore@gmail.com

\author{
Leandro Chernij \\ Universidade Tecnológica Federal do Paraná (UTFPR) \\ Av, Sete de setembro, 3165 - Curitiba - PR - Brasil \\ lchernij@gmail.com \\ Leonelo D. A. Almeida \\ Universidade Tecnológica Federal do Paraná (UTFPR) \\ Av, Sete de setembro, 3165 - Curitiba - PR - Brasil \\ leoneloalmeida@utfpr.edu.br
}

\begin{abstract}
Resumo A expansão da educação na modalidade à distância contribui sensivelmente à democratização do ensino. No entanto, o ensino de ciências experimentais ainda encontra diversas barreiras nessa modalidade como: a escassez de Ambientes Virtuais de Aprendizagem (AVAs) que oferecem recursos para essa prática de ensino, a baixa interatividade e o alto custo das plataformas existentes e a dependência de soluções comerciais. Esta pesquisa propõe o Apuê, uma plataforma web para a educação de ciências experimentais, construída somente com tecnologias livres e que provê alto nivel de interatividade, colaboração entre os estudantes, além de ser extensivel para o acoplamento de novos experimentos e flexivel na configuração e uso dos experimentos. Testes realizados com estudantes do ensino superior apontam que a plataforma atendeu e, frequentemente, superou as expectativas dos estudantes.
\end{abstract}

Palavras-Chave: educação a distância, ciências experimentais, laboratório remoto, ambiente virtual de aprendizagem, tecnologias livres

\begin{abstract}
The expansion of the modality of distance education contributes significantly to the democratization of education. However, the education of experimental sciences still faces several barriers in that modality as: lack of Virtual Learning Environments (VLEs) that provide features for that teaching practice, limited interactivity and high costs of the existing platforms, and the dependence on commercial solutions. This research proposes the Apuê, a web platform for education of experimental sciences, based only on free technologies and that provides high level of interactivity, collaboration among students, and is extensible for coupling new experiments and flexible for configuring and using the experiments. Tests involving undergraduate students indicate that the platform has met and, frequently, overcame the students' expectations.
\end{abstract}

Keywords: distance education, experimental sciences, remote laboratory, virtual learning environment, free technologies 


\section{Introdução}

A realização de atividades práticas, dentro de laboratórios, provou-se uma ferramenta desejável para o apoio do aprendizado de disciplinas como, por exemplo, a Física, a Química e a Biologia [17]. A interatividade e a dinâmica proporcionadas pelas atividades experimentais contribuem à atratividade das aulas tornando-as mais significativas aos estudantes e, consequentemente, resultando em um melhor aprendizado dos conceitos transmitidos durante as aulas teóricas. Dessa maneira, o estudante pode fazer a conexão dos conhecimentos teóricos previamente adquiridos com a prática, fato que pode ser associado com a aprendizagem significativa de Ausubel, onde os conhecimentos que serão adquiridos são associados a conhecimentos já existentes dos estudantes [2].

Partindo-se dessa perspectiva e contextualizando com a atual situação das instituições de ensino médio e superior no Brasil, podemos destacar alguns dos obstáculos encontrados pelas ciências experimentais: limitações de espaço físico dentro das instituições de ensino; escassez de recursos para aquisição e manutenção de laboratórios e equipamentos; e a quantidade limitada de profissionais capacitados para elaborar e conduzir as práticas laboratoriais [3].

A modalidade de Educação à Distância (EaD) é uma alternativa relevante para transpor as barreiras físicas, impostas pelas salas de aula e, também, incluir aqueles grupos sociais que, em condições normais, não teriam acesso à educação, por exemplo, pessoas economicamente desfavorecidas e habitantes de localizações distantes das instituições de ensino [14, 22]. A partir dos anos 90, com a ampliação do acesso à Internet, a EaD foi fortalecida pelo uso da computação para mediar as suas práticas e o desenvolvimento de Ambientes Virtuais de Aprendizagem (AVAs).

Entretanto, os AVAs ainda apresentam barreiras em determinadas modalidades de aula, uma delas são as práticas experimentais. Algumas pesquisas já propuseram plataformas para ciências experimentais remotas e que, no entanto, possuem limitações quando comparados às aulas experimentais presenciais (e.g. [24, 25]). Algumas das limitações relevantes são:

- Simulações virtuais dos experimentos: os resultados obtidos são sempre independentes dos fatores externos, que podem interferir nos experimentos reais, como umidade relativa do ar, temperatura ou erros operacionais [6];

- Ausência de uma plataforma consolidada e de fácil uso e, principalmente, baixo custo para aquisi- ção e manipulação de dados: conhecimentos avançados de eletrônica ou proficiência em programação não deveriam ser requisitos para docentes interessados em elaborar práticas laboratoriais. Dessa maneira, mais tempo seria investido na área de conhecimento do experimento e menos na configuração do ambiente;

- Uso de programas de terceiros para a interface da aplicação: dentre os trabalhos encontrados, todos fazem uso de programas de terceiros na implementação do programa cliente da solução, forçando o participante a adquirir ou instalar software;

- Interatividade limitada: soluções disponíveis apresentam baixa interatividade, o que empobrece a experiência do participante durante a experimentação e, consequentemente, impacta no processo de aprendizado como um todo.

Esta pesquisa propõe o Apuê, uma plataforma baseada na $W e b$ e tecnologias livres, para a realização de práticas laboratoriais - montadas em um espaço físico — de maneira que possam ser visualizadas, controladas e seus resultados analisados remotamente, independente da localização geográfica dos participantes das práticas. Este artigo apresenta o processo de concepção do Apuê, assim como um estudo de caso sobre o uso de um experimento sobre força centrípeta acoplado ao Apuê e utilizado por estudantes do ensino superior. O Apuê é uma extensão de um AVA e, além do experimento analisado no estudo de caso, provê funcionalidades para que novos experimentos possam ser adicionados a ele, sem a necessidade de codificação na plataforma.

O artigo está organizado da seguinte maneira: a seção 2 apresenta a revisão de literatura, que envolve a contextualização sobre as práticas laboratoriais e os trabalhos relacionados; a seção 3 apresenta o método de pesquisa e materiais empregados; a seção 4 apresenta a plataforma Apuê; a seção 5 apresenta o estudo de caso realizado utilizando um experimento sobre força centrípeta acoplado à plataforma Apuê e; a seção 6 apresenta as considerações finais do trabalho.

\section{Literatura relacionada}

Esta seção incialmente contextualiza as práticas laboratoriais e a sua relação com a EaD. Por fim é realizado o levantamento do estado-da-arte sobre plataformas computacionais para a educação de ciências experimentais. 


\subsection{Práticas laboratoriais e a EaD}

As atividades experimentais adquiriram importância no ensino de Ciências ao se configurarem como elementos essenciais para estudantes desenvolverem um aprendizado eficiente e estruturado. Neste formato de aula existe a possibilidade de utilizarem e manusearem, de maneira correta, os equipamentos e materiais; de presenciarem fenômenos químicos e físicos e organismos biológicos, além de terem a oportunidade de avaliar e obter suas próprias conclusões a partir dos resultados obtidos com as experiências realizadas.

Essas atividades contribuem de forma positiva ao desenvolvimento do estudante, por desenvolverem seu raciocínio e o interesse em solucionar problemas [3]. Além desses fatores, para Marineli e Pacca [17] a diferença entre as aulas teóricas das aulas nos laboratórios didáticos é por estar presente o referencial empírico, i.e., aquilo que é real, organizado especificamente para a experimentação, de maneira a interligar o conteúdo teórico com o experimento.

Mesmo com sua importância amplamente reconhecida, as aulas laboratoriais nem sempre são uma realidade no cotidiano dos estudantes, pois parte das escolas não possui materiais didáticos, seja por conta dos custos elevados e, até mesmo, espaço físico para preparar e montar os equipamentos referentes aos experimentos. Devido a essas barreiras, por vezes, professores ficam impelidos a utilizarem somente abordagens teóricas de aula, impossibilitando que os estudantes possam executar práticas empíricas para avaliar os conceitos apresentados em sala de aula [7].

Diante das barreiras encontradas para o desenvolvimento de práticas em laboratório, a utilização da modalidade de educação a distância, por meio de plataformas web para realização de experimentos, torna-se uma alternativa atrativa para possibilitar uma maneira de acesso às práticas laboratoriais.

A EaD tem sua origem datada desde muito antes da computação em rede. No Brasil a EaD passou por diversos meios de comunicação como, por exemplo, a Educação por Correspondência (EPC), a qual tem-se conhecimento, no país em 1904, através de representantes de instituições provenientes da América do Norte [4]. A Educação a Distância adaptou-se também ao rádio, tendo como uma das iniciativas o Projeto Minerva, o qual ocorreu no início da década de 70 e no curto período de um ano alcançou mais de 170 mil estudantes [18]. Também existiram iniciativas televisivas vinculadas à $\mathrm{EaD}$, dentre elas pode-se citar: os telecursos disponibilizados pelas fundações Bradesco, Padre Anchieta e Roberto Marinho [21].

Com o desenvolvimento de novas tecnologias e sua vinculação com a $\mathrm{EaD}$, surgiram diversas abordagens de ferramentas para dar suporte à EaD. Uma dessas ferramentas amplamente adotada pelas instituições de ensino são os Ambientes Virtuais de Aprendizagem (AVAs), que são sistemas computacionais disponíveis na Internet, mediados por Tecnologias Digitais de Informação e Comunicação (TDICs), e que proveem recursos para integração de diferentes mídias, linguagens e recursos, apresentação de informações, desenvolvimento de interações, produção e socialização de produções, independente do tempo e do espaço de cada participante [1].

Exemplos de AVAs disponibilizados sob licenças de código livre e amplamente adotados são: Aulanet [15], TelEduc, desenvolvido pelo NIED (Núcleo de Informática Aplicada à Educação) da Universidade Estadual de Campinas ${ }^{1}$; Moodle ${ }^{2}$; E-proinfo, desenvolvido pela Secretaria de Educação a Distância (SEED) do Ministério da Educação (MEC) ${ }^{3}$; e o Sakai Collaborative and Learning Environment (CLE) ${ }^{4}$.

A EaD mediada por AVAs tem se expandido no Brasil. Dados disponibilizados pelo Inep (Instituto Nacional de Pesquisas Educacionais Anísio Teixeira), no "Resumo Técnico Censo da Educação Superior de 2011" [8], apontam que as matrículas de graduação no ensino a distância apresentavam um número já superior a 990 mil em 2011. Referente aos tipos de cursos ofertados na modalidade da $\mathrm{EaD}$, foram encontrados os seguintes dados: $26,6 \%$ das matriculas são referentes a cursos tecnológicos, 30,2\% bacharelados e $43,3 \%$ licenciaturas.

\subsection{Plataformas para ciências experimentais}

Principalmente na última década, é possível encontrar pesquisas que tem o objetivo de propor ferramentas para a inclusão de práticas laboratoriais remotas na educação à distância mediada por TDICs (e.g. [24, 25]). Esta seção apresenta algumas soluções existentes relacionadas ao tema deste artigo e as categoriza diante de um conjunto de critérios que visam mitigar alguns dos problemas identificados nesta pesquisa e outros já relatados na literatura. Desse modo, é possível caracterizar as contribuições de cada solução e as oportunidades de investigação científica.

Os critérios utilizados para a análise comparativa da literatura consultada são:

- Uso de Aplicações Ricas para Internet: Faz referência ao uso, ou não, de aplicações ricas para Web na solução (e.g., Asynchronous Javascript and XML (AJAX), Java Applets, Flash e Hyper-

\footnotetext{
${ }^{1}$ http://www.teleduc.org.br/

${ }^{2} \mathrm{https}: / /$ moodle.org/about/

${ }^{3} \mathrm{http}: / /$ eproinfo.mec.gov

${ }^{4} \mathrm{https}: / /$ sakaiproject.org/
} 
Text Markup Language (HTML5)). Essa característica é relevante para plataformas de EaD pois, conforme citado na introdução do projeto, a interatividade afeta diretamente a experiência de aprendizado do participante;

- Sincronismo Cliente-servidor: Refere-se à capacidade da aplicação web enviar e receber dados de, ou para, um servidor web assincronamente, sem a necessidade de interferir no estado atual da interface do usuário. Essa funcionalidade pode oferecer uma experiência na $W e b$ mais interativa para o participante;

- Software Livre e Gratuito: Indica se a solução é composta inteiramente de software livre e gratuito em seu sistema, o que favorece a redução de custos e a extensibilidade da solução;

- Hardware Livre e Gratuito: Do mesmo modo que no item anterior, indica se todo o hardware utilizado no experimento é livre e gratuito;

- Transmissão de vídeo em tempo real: Critério sobre a disponibilização ao participante de transmissões de vídeo em tempo real do experimento que está sendo manipulado, o que propicia ao participante o acompanhamento visual e/ou auditivo da execução da prática dentro do laboratório;

- Foco em Extensibilidade: Este critério de comparação indica se a solução oferece recursos para, além de permitir o acoplamento de diferentes experimentos à plataforma subjacente (extensibilidade), abstrai o processo de estender a plataforma, minimizando o esforço e conhecimentos específicos necessários para tal, como programação e eletrônica;

- Padrões web: Indica se a plataforma web foi construída somente com o uso de tecnologias recomendadas pelo World Wide Web Consortium (W3C), tornando desnecessária a instalação, ou execução, de software de terceiros no cliente (e.g., Adobe Flash Player e Java Runtime Environment).

Para melhor apresentar as características e divergências intrínsecas aos projetos analisados, os dados estão dispostos sob o formato de um quadro comparativo (ver Tabela 1).

\begin{tabular}{|c|c|c|c|c|c|c|c|}
\hline Plataforma & $\begin{array}{c}\text { Aplicação } \\
\text { Rica }\end{array}$ & Sincronismo & Software & Hardware & Vídeo & $\begin{array}{l}\text { Extensi- } \\
\text { bilidade }\end{array}$ & $\begin{array}{c}\text { Padrões } \\
\text { web }\end{array}$ \\
\hline LabNet [24] & Sim & Assíncrona & Livre & N.D. ${ }^{1}$ & N.D. & Não & Sim \\
\hline $\begin{array}{l}\text { Desenvolvimentos em } \\
\text { hardware e software } \\
\text { para experimentos de } \\
\text { Física via Web [23] }\end{array}$ & Não & Síncrona & Proprietário & Livre & Não & Não & Sim \\
\hline ReLOAD [6] & Sim & Assíncrona & Proprietário & $\begin{array}{l}\text { Proprie- } \\
\text { tário }\end{array}$ & Sim & Sim & Sim \\
\hline FisL@bs [25] & Sim & Síncrona & Proprietário & N.D. & Sim & Sim & Não \\
\hline $\begin{array}{l}\text { Um ambiente para a } \\
\text { prática remota de } \\
\text { aulas laboratoriais } \\
\text { de Física [20] }\end{array}$ & Não & Assíncrona & Livre & N.D. & Sim & Não & Não \\
\hline WEBLAB [11] & N.D. & N.D. & N.D. & N.D. & Sim & Sim & N.D. \\
\hline Apuê & Sim & Assíncrona & Livre & Livre & Sim & Sim & Sim \\
\hline
\end{tabular}

Tabela 1: Tabela comparativa entre as plataformas existentes e o Apuê.

\footnotetext{
${ }^{1}$ Não Disponível
} 
É importante destacar que são poucos os trabalhos "Desenvolvimentos em Hardware e Software para Experimentos de Física" [23] e "Um Ambiente para a Prática Remota de Aulas Laboratoriais de Física" [20] — que se preocuparam explicitamente em garantir o baixo custo da solução final, por meio do uso de software gratuito e componentes físicos relativamente acessíveis. Porém, percebe-se que estes deixaram de explorar a comunicação assíncrona e aplicações ricas para Internet, o que potencialmente limita a interatividade entre a plataforma web e o participante ou, ainda, a comunicação entre os participantes. Uma alternativa para essas soluções seria, talvez, explorar os recursos introduzidos na primeira especificação completa do HTML5, introduzida em 17 de dezembro de $2012^{1}$. Assim, por se tratar de uma tecnologia ainda incipiente, esses trabalhos não puderam usufruir de seus recursos, pois o HTML5 não estava disponível no momento em que foram desenvolvidos.

Outro destaque a ser feito é referente às soluções ReLOAD [6] e FisL@bs [25], que fizeram uso de uma solução comercial chamada LabView ${ }^{2}$, uma ferramenta que facilita a integração de sistemas com hardware, possibilitando seu controle e medições. Entretanto, a ferramenta tem um custo significativo ${ }^{3}$, encarecendo o custo de aquisição ou reprodução da solução em instituições de ensino públicas. Portanto, apesar desses trabalhos baseados no LabView terem adicionado funcionalidades relevantes como a extensibilidade da plataforma, o custo de aquisição da plataforma torna-se uma barreira a sua ampla adoção.

Por fim, os trabalhos publicados do LabNet [24] e WEBLAB [11] carecem de detalhes de implementação e desenvolvimento, pois as poucas informações disponíveis tornam difícil a análise de aspectos como custo e licenças de hardware; consequentemente a análise das plataformas fica debilitada.

Outros dois trabalhos recentes também abordam o tema. Guimarães et al. [5] propuseram uma prática laboratorial de uma experiência envolvendo um pêndulo, o qual foi totalmente configurado para ser controlado remotamente, através de uma página web e que inclui transmissão de vídeo. Porém essa proposta é voltada somente para esse experimento, não sendo extensível a outros. O segundo trabalho, de Lucena, Santos e Silva [16], propõe um programa de simulação de um laboratório de química para apresentar as reações químicas e outras peculiaridades que ocorrem em laboratórios. Ambos os trabalhos, apesar de soluções interessantes, não atendem plenamente os critérios considerados nesta pesquisa e apontados na

\footnotetext{
${ }^{1}$ http://www.w3.org/TR/html5/

${ }^{2}$ www.ni.com/labview/

${ }^{3}$ Custo de $\$ 1100$ (um mil e cem dólares) pela versão LabVIEW Base e mais de \$5400,00 (cinco mil e quatrocentos dólares) pela versão LabVIEW Professional. Consulta realizada em abril de 2014.
}

última linha da Tabela 1, a plataforma Apuê.

\section{Materiais e métodos}

O método de desenvolvimento do projeto consistiu de seis atividades realizadas de maneira iterativa e não sequencial: (1) levantamento de requisitos, (2) seleção de um experimento para instanciação na plataforma, (3) definição da plataforma tecnológica, (4) adaptação do hardware do experimento para acoplá-lo à plataforma, (5) desenvolvimento da plataforma e (6) estudo de caso.

\subsection{Participantes do projeto}

O Apuê está inserido no contexto de um projeto de fomento à criação de recursos educacionais abertos, financiado pela PROGRAD/UTFPR. O desenvolvimento do projeto foi realizado por uma equipe de 5 pessoas, sendo três delas bolsistas do projeto, um voluntário e o coordenador do projeto.

O levantamento de requisitos, além de utilizar a revisão de literatura, envolveu a aplicação de um questionário a professores e estudantes das áreas científicas, além de entrevistas presenciais com professores de Física (UTFPR, Curitiba) e uma professora do ensino médio. Ao todo, a pesquisa envolveu 63 participantes, sendo 26 estudantes do ensino médio presencial, 14 estudantes do ensino médio a distância, 20 estudantes do ensino superior e 3 professores, sendo um mestre e dois doutores. As entrevistas com os professores envolveram tanto os requisitos para a plataforma quanto sugestões de experimentos que pudessem ser acoplados a ela. Todos os participantes/responsáveis foram informados e assinaram um Termo de Consentimento Livre e Esclarecido (TCLE).

O estudo de caso para a avaliação da plataforma Apuê teve a participação de 8 estudantes do ensino superior da UTFPR/Curitiba. A dinâmica de avaliação utilizada foi a de uma aula experimental. Cada seção de aula teve a participação 2 estudantes, sendo essa divisão feita para simular o uso da plataforma por dois usuários simultaneamente. $\mathrm{O}$ número de participantes por sessão escolhidos deveu-se à limitação de pesquisadores para conduzir e coletar os dados da atividade. Inicialmente um pesquisador, no papel de apresentador, descreveu a plataforma e os conceitos de Física envolvidos no experimento. Em seguida foram distribuídos questionários pré-teste e os TCLEs. Após esta etapa os estudantes executaram os testes em duplas e foram auxiliados por pesquisadores, quando demonstravam estar com dificuldades. Durante a sessão do experimento, o papel de administrador/professor da plataforma Apuê foi assumido por um pesquisador. Este poderia delegar o controle da plataforma aos participantes da sessão a qualquer momento. Ao final dos testes cada estudante recebeu um questionário 
pós-teste para avaliar o uso do Apuê. Durante a realização dos testes foi utilizado um software para gravação de telas. Outro pesquisador esteve responsável por coletar fotos e trechos de vídeos relevantes.

\subsection{Plataforma tecnológica}

A plataforma Apuê foi projetada em três camadas: a

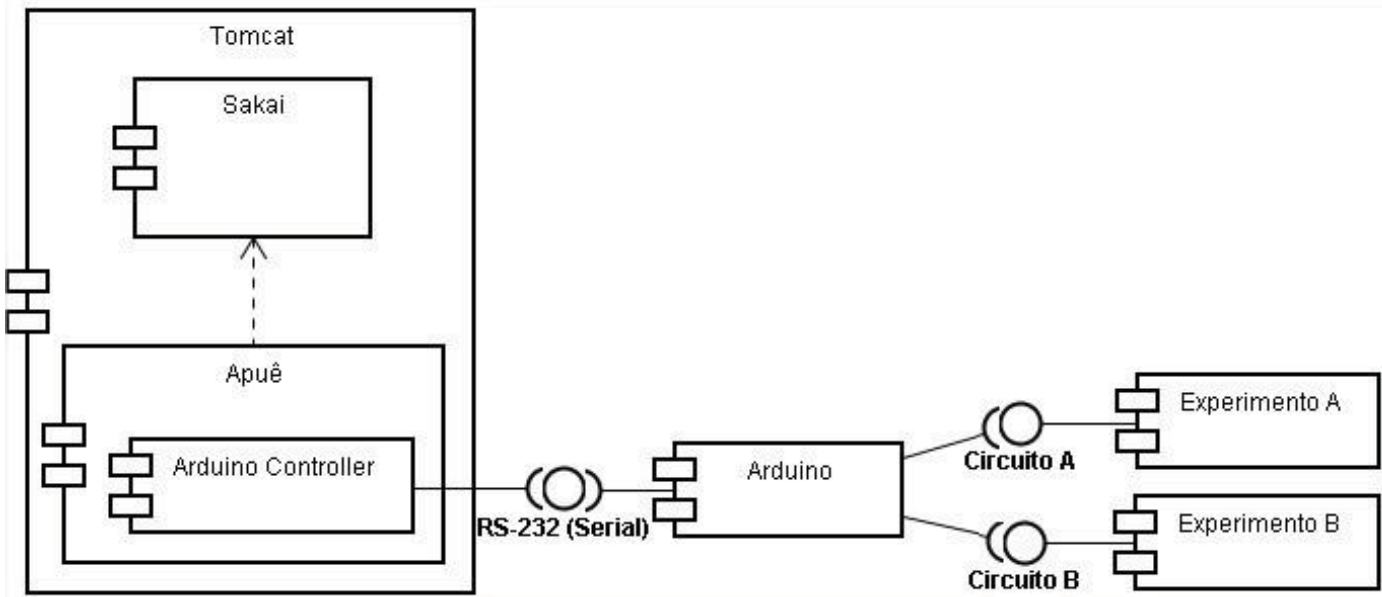

Figura 1: Diagrama de componente de integração do Apuê e do controlador do Arduino (middleware) com o experimento.

A camada web foi desenvolvida como uma extensão de um AVA existente, o Sakai Collaboration Learning Environment (CLE). O Sakai é um AVA livre que conta com uma extensa base de usuários (quatro milhões de participantes) e uma comunidade de desenvolvimento ativa. A principal vantagem em se usar um AVA existente é evitar o desenvolvimento de uma grande quantidade de funcionalidades, comumente encontradas em sistemas de $\mathrm{EaD}$, que não fazem parte do escopo do projeto (e.g., controle de usuários, avaliações, fóruns de discussões, calendários acadêmicos, compartilhamento de arquivos, entre outras).

A plataforma web (chamada Apuê Web) é o ponto de entrada para os participantes dos experimentos remotos. Ela é responsável pelo controle de acesso de usuários, o agendamento de aulas de laboratório e o provimento de recursos para que estudantes e professores possam reservar horários para a utilização dos experimentos. Toda a interface preza pelas tecnologias padrão da $W e b$, definidas pelo $\mathrm{W} 3 \mathrm{C}$, inclusive a transmissão do vídeo exibido ao participante, em tempo real, da experimentação. A plataforma é construída sobre o framework JEE (Java Platform, Enterprise Edition) e é integrada com uma camada controladora do Arduino.

O Arduino é uma solução de prototipagem de baixo custo, baseada em hardware livre, para a criação de ferramentas mais acessíveis, flexíveis e de fácil uso. Ele é voltado para o público com pouco conhecimento na área de desenvolvimento de protótipos eletrônicos. Neste plataforma web (interface para interação de professores e estudantes), o controlador Arduino (middleware) e a plataforma de hardware (os experimentos). A Figura 1 representa o diagrama de componentes da integração realizada no projeto. projeto, o Arduino foi utilizado como uma ponte entre a plataforma Apuê e os componentes físicos dos experimentos.

Para que a transmissão de vídeo dos experimentos fosse possível, foi utilizado o framework multimídia FFMPEG $^{1}$ para captar o vídeo de câmeras e gravar os experimentos; e Node.js ${ }^{2}$ com WebSocket $^{3}$ para disponibilizá-los na $W e b$, em tempo real ou sob a demanda do usuário.

Para a persistência dos dados da solução é utilizado o Sistema Gerenciador de Bancos de Dados (SGBD) gratuito e de código aberto, MySQL ${ }^{4}$. Apesar de não ser um dos SGBDs mais sofisticados, o MySQL é um SGBD relacional leve, de configuração relativamente simples e de alta performance. O MySQL é, também, um dos SGBDs mais frequentemente suportados pelos AVAs, assim como o PostgreSQL ${ }^{5}$.

No Apuê, o usuário faz requisições que passam por uma camada controladora do Arduino que, até certo ponto, atua como um software middleware ${ }^{6}$. Em seguida, estas informações são repassadas ao Arduino que, por sua

\footnotetext{
${ }^{1}$ http://www.ffmpeg.org/

${ }^{2} \mathrm{http}: / /$ nodejs.org/

${ }^{3} \mathrm{http}: / /$ www.websocket.org/

${ }^{4}$ http://www.mysql.com

${ }^{5} \mathrm{http}: / /$ www.postgresql.org

${ }^{6}$ Trata-se de um sistema que possibilita o trabalho em conjunto de diferentes dispositivos e softwares (Encyclopcedia Britannica, acesso em 2013)
} 
vez, controla o experimento e retorna respostas ao middleware. As respostas são processadas e apresentadas aos usuários. Esta organização permite o isolamento da comunicação, pois todo o processamento dos dados obtidos é feito no middleware, além de que os resultados deste processamento podem ser facilmente distribuídos entre os diversos usuários que estarão acompanhando o experimento.

A biblioteca RXTX $^{1}$ é utilizada para a comunicação entre a plataforma Java, utilizada no middleware e a placa Arduino, através da porta serial, utilizando o protocolo RS-232. Esta biblioteca foi escolhida por ser um software livre, que pode ser redistribuído ou modificado sob os termos da GNU Lesser General Public License [9], por possuir também a capacidade de se comunicar com a Integrated Development Environment (IDE) do Arduino, além de ser oficialmente documentada pela comunidade do Arduino ${ }^{2}$.

A coleta de dados da prática laboratorial — por exemplo, a velocidade de um objeto, a distância percorrida, o tempo decorrido e outros - é realizada por meio de componentes eletrônicos (e.g., sensores e motores) interligados aos pinos de entrada e saída do Arduino. Este, por meio de uma porta serial, envia os dados recebidos para o middleware, por meio de um programa baseado na biblioteca RXTX.

De maneira análoga, dados podem ser enviados do middleware para o Arduino permitindo, por exemplo, ligar e desligar um sensor ou motor, aumentar ou diminuir a rotação de um determinado motor.

\subsection{Experimento sobre Força Centrípeta}

O experimento selecionado, com o apoio dos professores do departamento de Física/UTFPR, foi a plataforma rotacional para estudo do movimento circular e suas variáveis (ver Figura 2). A plataforma foi desenvolvida com o objetivo de estudar a dinâmica rotacional, mais precisamente a segunda lei de Newton aplicada à dinâmica rotacional.

A principal equação que se pode estudar com essa plataforma é a equação da força centrípeta, que é a força resultante que puxa o corpo para o centro da trajetória em um movimento curvilíneo ou circular. A plataforma contempla não só o estudo da força centrípeta, mas sim toda a dinâmica rotacional, como: momento de inércia, velocidade angular, aceleração angular, torque, entre outros. Para isso, basta fazer pequenas modificações e inserir outros módulos.

O módulo a ser estudado nesta configuração do experimento envolve a força centrípeta, que consiste em esco-

\footnotetext{
${ }^{1}$ http://rxtx.qbang.org/wiki/index.php/Main_Page

${ }^{2} \mathrm{http}: / /$ playground.arduino.cc/Interfacing/Java
}

lher a massa que vai orbitar (massa orbitante "M", na Figura 2) e escolher a massa que vai deixar suspensa para tracionar a mola (massa de comparação com a força centrípeta, indicada por massa suspensa “m”, na Figura 2).

A análise a ser produzida será a de Força centrípeta X Aceleração centrípeta. Neste caso sabe-se que o valor da força centrípeta será o mesmo da massa que será pendurada pelo fio de nivelamento (aquele ligado à pastilha de nivelamento) multiplicado pelo valor da gravidade. Chega-se a essa conclusão devido a "Pastilha de Nivelamento" estar previamente ajustada sobre a influência da massa em questão (massa suspensa), tal como representado pela Figura 2.

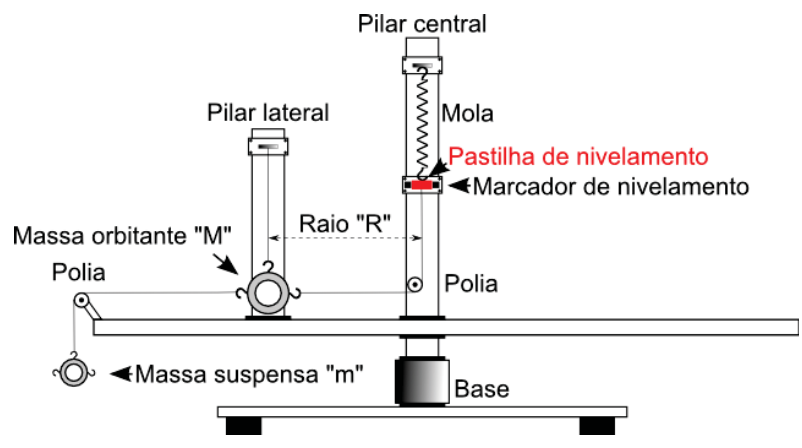

Figura 2: Ilustração da plataforma rotacional na configuração inicial (com a massa suspensa).

Em um segundo momento, a massa suspensa é retirada do experimento (ver Figura 3). Com isso a mola reduz a sua deformação e a pastilha de nivelamento deixa de estar alinhada ao marcador de nivelamento. Ainda, a massa orbitante é puxada pela mola na direção do pilar central. Em seguida, a plataforma é colocada para girar. No momento em que a pastilha de nivelamento atingir o ponto anterior (alinhada com o marcador de nivelamento), a força centrípeta será igual ao peso da massa.

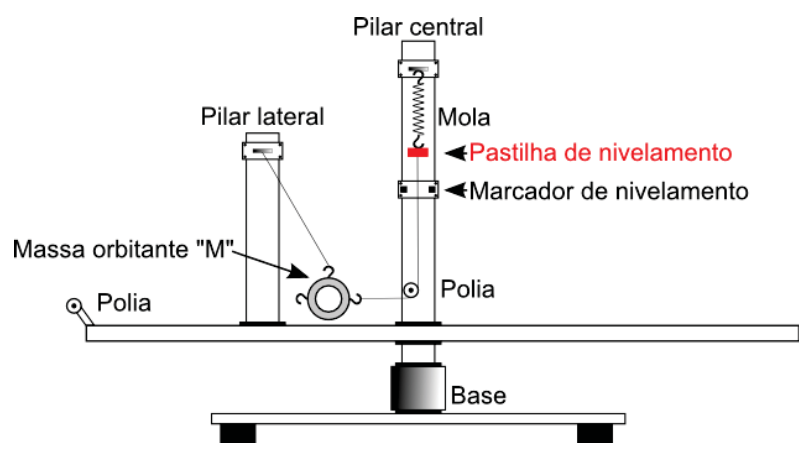

Figura 3. Ilustração da plataforma rotacional sem a massa suspensa.

Durante o experimento são utilizadas principalmente duas equações, derivadas da segunda lei de Newton, para determinar a força centrípeta (ver Equação 1) e a acelera- 
ção centrípeta (ver Equação 2).

$$
\begin{gathered}
\mathrm{Fc}=\mathrm{m} * 4\left(\frac{\pi}{\mathrm{T}}\right)^{2} * \mathrm{R} \\
\mathrm{Ac}=4\left(\frac{\pi}{\mathrm{T}}\right)^{2} * \mathrm{R}
\end{gathered}
$$

A Equação 1 representa a magnitude da força que está puxando a massa orbitante $m$ em direção ao pilar central, quando a plataforma está em movimento. A Equação 2, por sua vez, representa a taxa de variação da velocidade dessa massa ao longo do tempo. Em ambas $T$ representa o tempo de um ciclo da plataforma e $R$ o raio entre o pilar central e o pilar lateral (indicado na Figura 2).

\section{A plataforma Apuê}

A apresentação da plataforma Apuê compreende a lista de requisitos oriundos da atividade de levantamento, a adaptação do experimento de força centrípeta à plataforma e seu acoplamento à plataforma, e os componentes do módulo Web da plataforma Apuê e sua integração ao AVA Sakai.

\subsection{Requisitos e modelagem}

O projeto da plataforma teve seu início com o levantamento de requisitos realizado por meio da aplicação de questionários a estudantes do ensino médio e superior, do ensino presencial e a distância e de professores de Física, além da funcionalidades das plataformas analisadas na revisão de literatura.

A lista resumida de requisitos identificados é apresentada a seguir.

Requisitos não funcionais:

- RNF1 Baixo custo da solução final: a plataforma deve exigir poucos recursos financeiros da parte interessada;

- RNF2 Qualidade de vídeo: a qualidade final da transmissão de vídeo do experimento deve ser suficiente para permitir a visualização nítida pelos participantes do sistema;

- RNF3 Independência de software adicional: o uso da plataforma não deve exigir a instalação de software adicional no computador do usuário;

- RNF4 Tecnologias livres: todos os componentes da plataforma devem ser livres;

- RNF5 Extensibilidade: a plataforma deverá ser extensível, permitindo acoplamento de outros experimentos.
Requisitos Funcionais:

- RF1 Visualizar o vídeo em "tempo real” do experimento remotamente;

- RF2 Controlar o experimento remotamente: por meio de uma interface de usuário gráfica, alterar o estado e o comportamento do experimento;

- RF3 Criar sessão de experimento: permitir acesso simultâneo a um grupo de participantes;

- RF4 Controlar sessão de experimento: prover recursos para que um participante possa controlar o experimento e delegar esse controle a outro participante, enquanto os demais o visualizam;

- RF5 Visualizar os dados do experimento: o sistema deverá prover aos participantes de uma sessão de experimento, dados que estão sendo coletados nos componentes eletrônicos (e.g., sensores infravermelho, sensores de luminosidade, giroscópio) do mesmo;

- RF6 Funcionalidades comuns a AVAs como: autenticação de participantes; blog, calendários compartilhado de atividade; chat, fóruns de discussão, compartilhamento de arquivos, glossário, notícias, organização e armazenamento de materiais, provas e questionários.

A partir dos requisitos foi realizada a modelagem da plataforma. Para tanto, foram utilizados diagramas de caso de uso, diagramas de sequência, diagramas de componentes e diagramas de classes. Por limitações de espaço, este artigo apresenta somente o diagrama de classes da camada de modelo (ver Figura 4) e o diagrama de componentes, já apresentado na Figura 1.

A camada de modelo do diagrama de classes contém todas as classes que modelam os dados da aplicação Apuê e, na sua maioria, representam entidades Java Persistence API (JPA) ${ }^{1}$, que traduzem-se em tabelas do banco de dados. As entidades JPA e suas operações básicas foram geradas a partir do banco de dados, por engenharia reversa.

No Apuê, a classe Experimento representa os experimentos que estão acoplados à plataforma. A classe Metadado representa as descrições dos dados, de entrada e de saída, envolvidos no experimento. MetadadoEntrada e MetadadoSaida, representam os metadados cadastrados que serão enviados ao experimento e os que o experimento enviará para a plataforma, respectiva-

\footnotetext{
$1 \mathrm{http} / /$ www.oracle.com/technetwork/java/javaee/tech/persistence-jsp140049.html
} 
mente. As classes TipoEntrada e OpcaoMetadadoEntrada são empregadas para definir o formato de apresentação dos metadados de entrada, na interface de usuário para interação com o experimento. A classe Gráfico contém os dados relacionados aos gráficos que serão exibidos durante as sessões de uso do experimento. Para que experimentos possam ser manipulados por meio da plataforma Apuê, é necessário realizar um agendamento de sessão de uso, representado na classe Sessao. As classes Sessaogrupo, SessaoGrupopk, Dado, TipoDado e UpdateSessao são utilizadas para controlar os grupos de participantes que podem acessar a sessão de uso e os dados trocados entre o experimento e a plataforma Apuê.

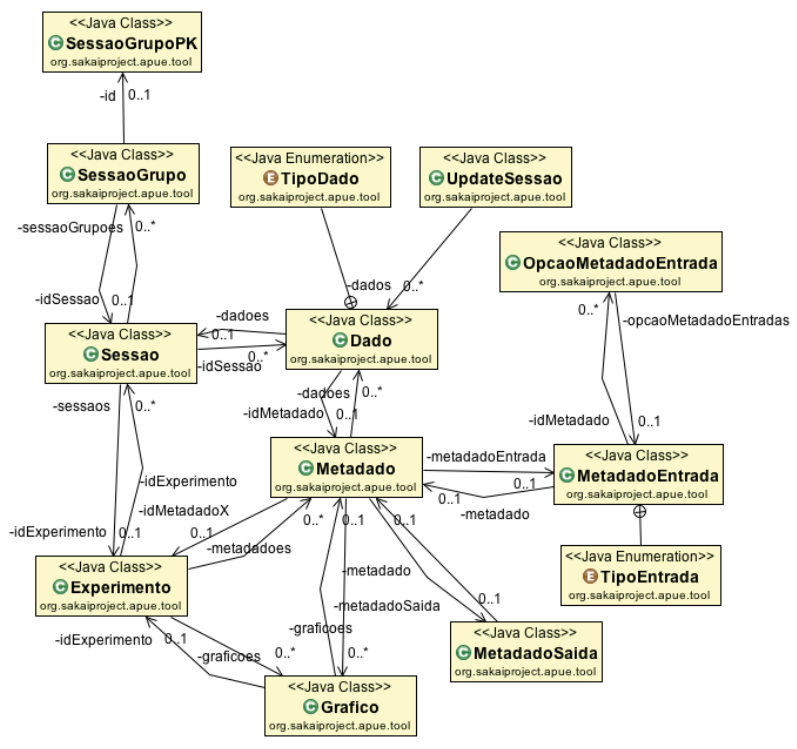

Figura 4: Diagrama de classes da camada modelo da plataforma Apuê.

\subsection{Adaptação do experimento}

O experimento apresentado na Figura 5 foi cedido pelo Departamento de Física da UTFPR/Curitiba. Características relevantes sobre os componentes deste experimento são o uso de materiais de baixo custo e a flexibilidade para ajustes e adições de novos componentes. Nesta figura o experimento se encontra na configuração inicial, ou seja, com a massa suspensa presente (i.e. Massa “m”). Também é possível identificar os elementos cronômetro e caixa de controle. Para a integração com o Apuê, esses dois últimos elementos foram substituídos e passaram a ser controlados pelo Arduino.

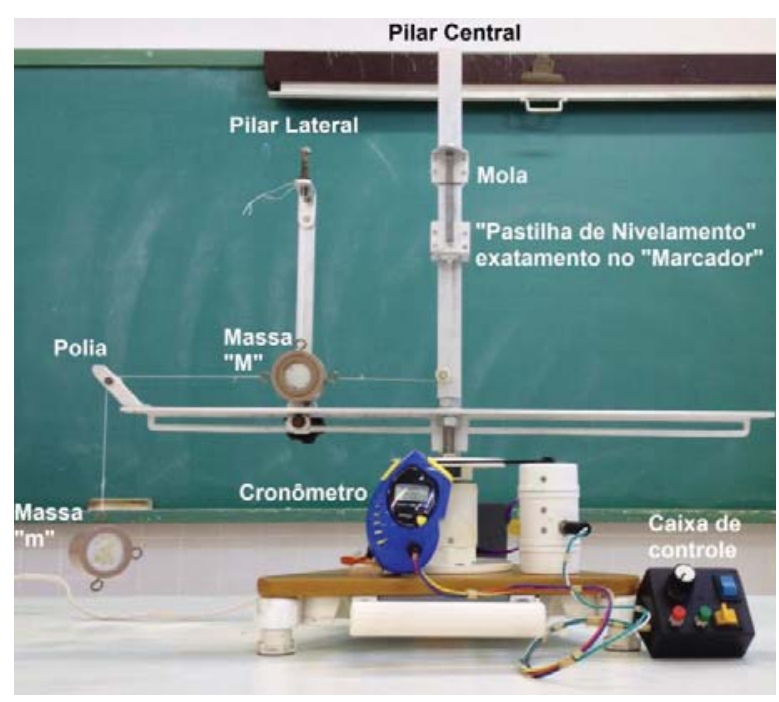

Figura 5: Plataforma rotacional.

Para a integração do experimento ao Arduino foram utilizados componentes físicos e de software. Entre os componentes físicos estão: ponte H L293D (um circuito integrado que serve para controlar o sentido e a intensidade do funcionamento dos motores de corrente contínua (DC); resistor de 10K Ohms (para limitar a corrente da fonte para o circuito); conector P2 fêmea e macho (para conectar o sensor de rotação); Arduino Protoboard Shield (para acomodar os fios, conectores, a ponte $\mathrm{H}$ e o resistor); motor DC de 9V (pertencente ao experimento); e Reed Switch (também pertencente ao experimento).

Para realizar o envio dos comandos recebidos da camada de comunicação para o experimento e para enviar os dados coletados do experimento para a camada de comunicação foi criado um protocolo utilizando as notações JSON (JavaScript Object Notation) (para a plataforma web) e aJSON (para o Arduino [19]). O protocolo consiste nas etapas:

- Plataforma web para Arduino: A plataforma web envia um objeto JSON contendo o nome da chave do dado de entrada (e.g., velocidade) e o valor (e.g., 15,2) que iria atribuir a essa chave, o qual é recebido pelo aJSON, através da porta serial, e decodifica o objeto para ser utilizando no código do Arduino, que por fim passa os valores para os respectivos componentes do sistema;

- Arduino para plataforma web: O Arduino recebe os dados dos componentes do experimento, os empacota em objetos JSON e os envia através da porta serial para a plataforma web, a qual exibe para o usuário os dados referentes a saída. 


\subsection{Plataforma web}

O plataforma web foi desenvolvida como um módulo do Sakai, chamado Apuê Web. O AVA Sakai faz uso da ferramenta Maven ${ }^{1}$ para automação do processo de compilação, empacotamento e distribuição. A estrutura básica do Apuê Web utiliza o maven archetype criado por Jennings [10] para gerar um módulo Sakai, com Spring $\mathrm{MVC}^{2}$ como framework web. Posteriormente o Apuê $W e b$ foi modificado para se adequar aos padrões introduzidos no Spring MVC 3. Entre elas, alterações de bibliotecas, arquivos de configuração e o formato de injeção de dependências de XML para Java annotations.

As principais funcionalidades disponíveis aos usuários pela plataforma web são: (1) configuração de experimentos, (2) agendamento de sessões e (3) visualização e controle de experimentos. Apesar da diversidade de tecnologias empregadas na construção da plataforma Apuê, para o usuário da plataforma basta ter um navegador web convencional (e.g. Mozilla Firefox, Google Chrome, Microsoft Internet Explorer) e não há necessidade de instalação de nenhuma extensão de software.

Configuração de experimentos. Esta funcionalidade permite aos professores adicionar, alterar ou remover experimentos. Para facilitar o uso desta funcionalidade, a interface de usuário foi dividida em etapas: dados gerais do experimento, dados de entrada, dados de saída e gráficos. Na primeira etapa o professor visualiza a lista de experimentos cadastrados e pode escolher a operação desejada (i.e. criação, alteração e remoção). Para a criação ou alteração de experimentos, os dados disponíveis para serem informados, em etapas, são:

- Dados gerais do experimento: nome do experimento, descrição do experimento, Unified Resource Locator (URL) onde está disponível o WebSocket do vídeo do experimento, porta Universal Serial Bus (USB) em que o experimento está conectado ao servidor;

- Dados de entrada (são os dados que serão enviados da plataforma web para o experimento): chave (nome interno para o protocolo de troca de dados entre o Arduino e a plataforma web), rótulo (nome a ser exibido aos usuários durante a sessão de experimento), descrição do dado, unidade de medida do dado (e.g. metros/segundo, centímetros), visibilidade (em relação à área de saída de dados da

\footnotetext{
${ }^{1} \mathrm{http}: / /$ maven.apache.org

2 É um framework de código aberto desenvolvido para aplicações web Java baseado no modelo "Model-View-Controller" com o intuito de simplificar o desenvolvimento e os testes das aplicações. http://docs.spring.io/spring/docs/3.0.x/reference/new-in-3.html
}

funcionalidade de visualização e controle de experimentos), valor inicial padrão, tipo do elemento (elemento HTML para informação do dado e.g. seleção simples, múltipla escolha, entrada de texto). Quando apropriado, também deverão ser informados os valores possíveis (e.g. "sim/não" para uma seleção simples);

- Dados de saída (são os dados que serão enviados do experimento para a plataforma web): chave, rótulo, descrição e unidade. O propósito destes dados é o mesmo que os dos dados de entrada;

- Gráficos: Nesta etapa o professor pode cadastrar quantos gráficos desejar para o experimento. Para tanto deve informar o dado de saída que será usado no eixo vertical. No eixo horizontal estão as marcas de tempo.

Agendamento de sessões. A funcionalidade de agendamento de sessões é bastante simples. O usuário tem a opção de criar um novo agendamento, alterar ou remover um agendamento existente. As informações necessárias para um agendamento são: experimento que será utilizado, grupo de participantes (a funcionalidade de manutenção de grupos é fornecida pelo Sakai) e as datas e horas de início e fim da sessão. Inicialmente, somente professores tem acesso ao agendamento de sessões.

Visualização e controle de experimentos. Esta é uma funcionalidade dinâmica, construída a partir do cadastro do experimento e sem a necessidade de codificação pelo usuário. A funcionalidade de visualização e controle de experimentos reúne diversos recursos, tal como destacado na Figura 6: (1) visualização do vídeo do experimento; (2) gráficos dinâmicos dos dados oriundos do experimento (construído com a biblioteca HighStock $^{3}$ ); (3) visualização de qual participante está com o controle do experimento e a possibilidade de delegar o controle a outro participante (some visível a quem tem o controle atualmente e ao professor responsável pela sessão); (4) dados de entrada (i.e., os dados que serão enviados ao experimento, de maneira a alterar seu estado); e (5) dados de saída (i.e., os últimos dados gerados pelo experimento). Durante uma sessão há sempre um professor e diversos alunos, sendo que um destes alunos pode assumir o controle do experimento, enquanto os demais observam os eventos que estão ocorrendo nos elementos denotados pela Figura 6. Tanto o aluno com o controle do experimento quanto o professor podem delegar o controle do experimento aos demais alunos, sendo que apenas um aluno pode assumir o controle por vez.

\footnotetext{
${ }^{3}$ http://www.highcharts.com/products/highstock
} 
Força Centripeta

Controlador $\quad 3$ labadmin1 0 Delegar
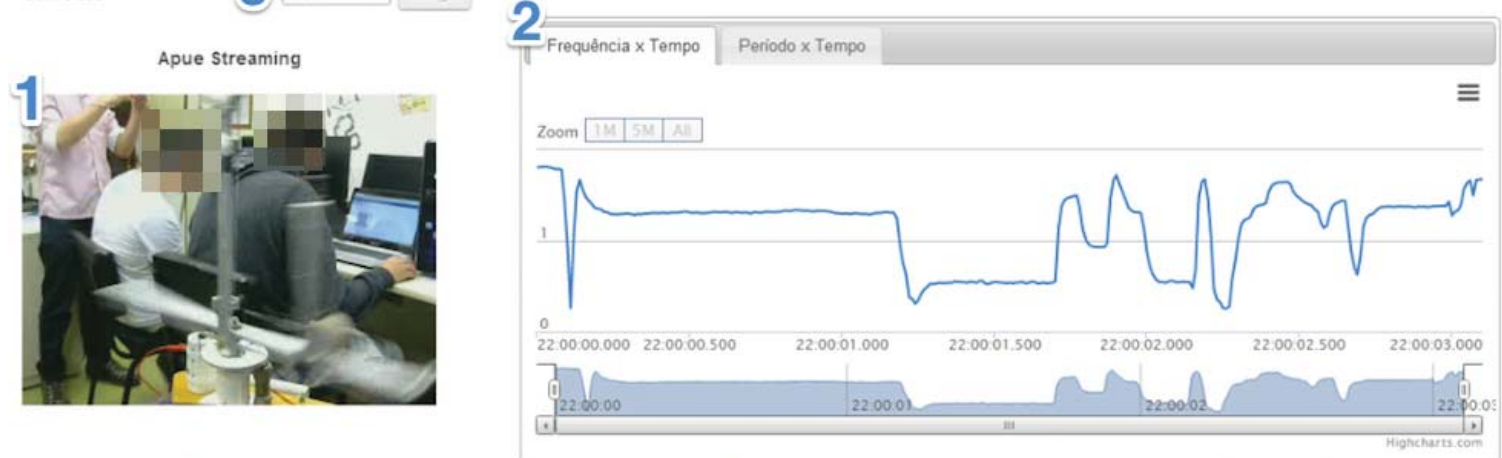

Dados de Entrada 4

Velocidade do Motor

4

Foncernerios

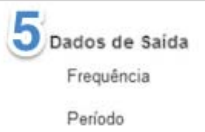

Frequência

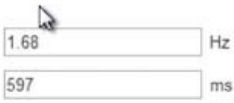

$\mathrm{Hz}$

Figura 6: Interface para visualização e controle de experimentos.

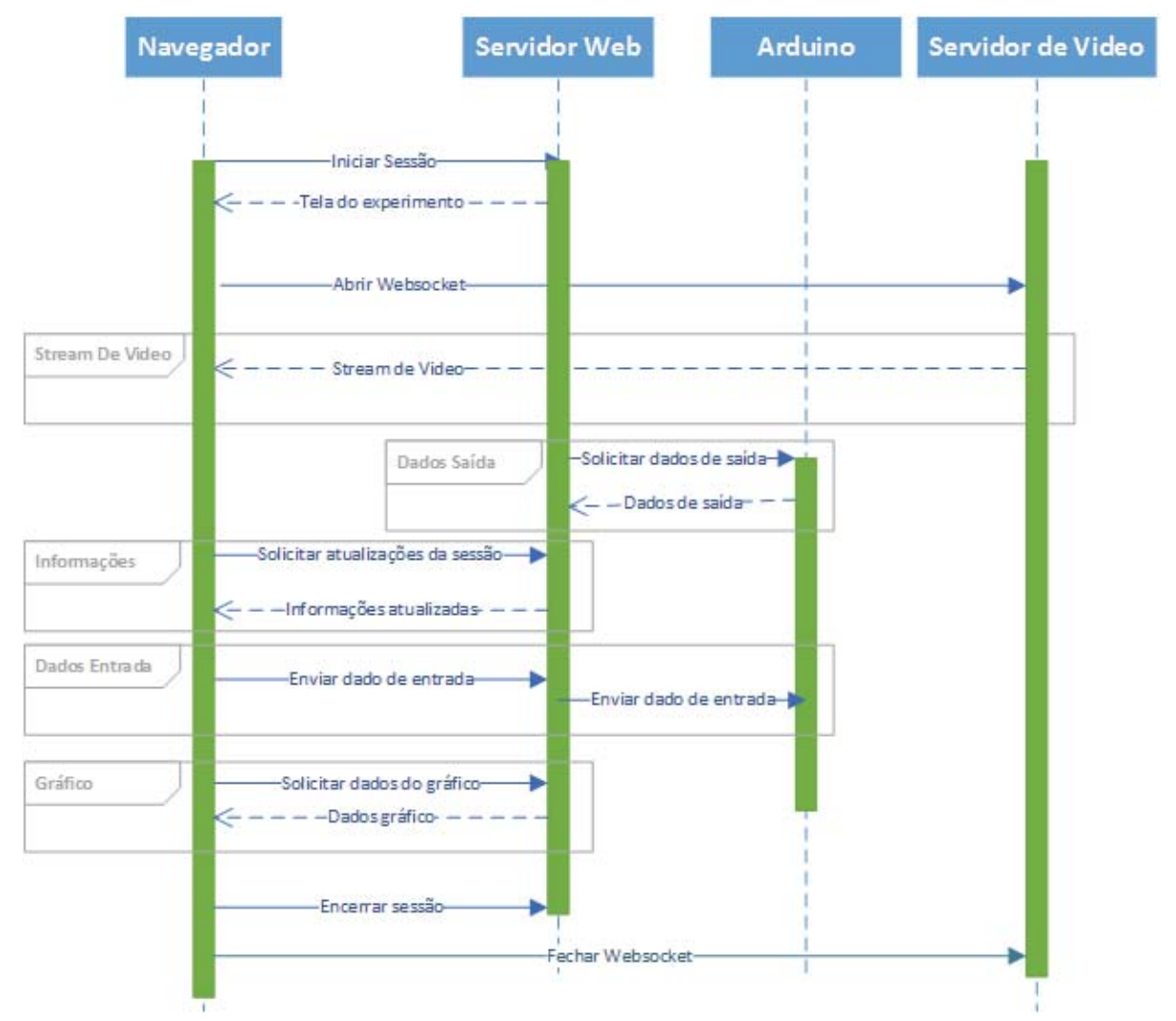

Figura 7: Diagrama de sequência de sistema da funcionalidade de visualização e controle de experimentos. 
Assim que o participante entra no módulo Apuê, o módulo verifica se há um agendamento de sessão para aquele momento em que o participante esteja incluído. Em caso positivo inicia-se o processo de carregamento do experimento. Nessa etapa são recuperadas do banco de dados, as informações referentes ao experimento cadastrado, necessárias para a execução da sessão, e.g., metadados de entrada e de saída, URL do WebSocket para o streaming do vídeo e gráficos.

No momento em que as informações do experimento são recuperadas e o usuário recebe a página principal, inicia-se o processo de carregamento assíncrono dos dados da sessão, via AJAX. Em paralelo, o WebSocket de streaming é aberto e conectado ao servidor Node.js, permitindo o início da renderização do vídeo (indicado pelo item 1, na Figura 6) pela biblioteca JSMpeg, dentro da tag HTML canvas $^{1}$, seguindo a recomendação HTML5 ${ }^{2}$. As requisições AJAX são temporizadas (invocadas a cada 1,5 segundos), permitindo a atualização quase instantânea dos dados exibidos - técnica conhecida também como "atualização AJAX periódica" [12].

A dinâmica das mensagens trocadas entre o navegador web do computador do estudante/professor com os diversos componentes da funcionalidade de visualização e controle de experimentos está representada pelo diagrama de sequência de sistema na Figura 7.

Com o propósito de evitar o envio de muitas informações em uma única requisição, a interface é atualizada por dois grupos de invocações AJAX:

- Atualização dos dados da sessão: responsável por receber as últimas alterações no usuário controlador (item 3 na Figura 6), dados de entrada (item 4) e dados de saída (item 5);

- Atualização dos dados nos gráficos: para cada gráfico (item 2) cadastrado no experimento é periodizada uma chamada AJAX, responsável por buscar atualizações de novos valores que serão adicionados na série do respectivo gráfico.

\section{Estudo de caso}

O estudo de caso realizado envolveu a aplicação de questionários pré-teste e pós-teste, a exploração da plataforma Apuê por pares de participantes, as anotações feitas pelos pesquisadores durante a exploração e gravação das telas dos participantes durante a exploração. Por fim,

\footnotetext{
${ }^{1}$ Uma área que é um mapa de bits dependente de resolução que pode ser usado para renderizar grafos, gráficos e outras imagens em tempo de execução.

${ }^{2}$ http://www.w3.org/TR/html5/
}

esta seção apresenta uma discussão sobre os resultados obtidos pela pesquisa.

\subsection{Resultados}

Após a explicação inicial do objetivo do estudo de caso e do TCLE, os participantes foram convidados a responder um questionário pré-teste. $\mathrm{O}$ objetivo deste questionário foi entender a experiência prévia dos participantes com este tipo de ferramenta e de analisar as expectativas dos participantes em relação à plataforma Apuê. As perguntas foram construídas usando a escala Likert [13] e algumas delas tinham a opção de complemento textual.

As perguntas envolveram: a opinião dos estudantes em relação às aulas laboratoriais no ensino da Física; a experiência prévia dos estudantes com AVAs, se os AVAs utilizados proviam recursos para práticas laboratoriais remotas e a opinião do participante sobre a relevância deste tipo de ferramenta; por fim, as expectativas e opiniões dos participantes, em relação a algumas das funcionalidades da plataforma proposta.

Em relação à relevância das práticas laboratoriais, $87,5 \%$ dos participantes informaram que as atividades laboratoriais são essenciais para o ensino/aprendizado, enquanto que $12,5 \%$ acreditam que estas atividades são relevantes no ensino. Sobre a experiência prévia com AVAs, $50 \%$ dos participantes informaram já terem usado algum AVA, sendo o AVA Moodle o mais comum. Porém nenhum participante afirmou ter usado um AVA com funcionalidades para práticas laboratoriais remotas.

Quando questionados sobre as expectativas em relação ao uso de uma plataforma para práticas laboratoriais remotas, $87,5 \%$ dos participantes informaram a expectativa de que a plataforma fosse de fácil utilização e, somente $12.5 \%$, esperavam que o uso e compreensão não seriam tão simples. A maior parte dos participantes (75\%) apontou que a visualização do vídeo seria essencial ou muito importante para o aprendizado, enquanto que $25 \%$ dos participantes não responderam esta pergunta. Já, em relação aos gráficos dos dados de saída, 87,5\% dos participantes tinham a expectativa que a construção em tempo real seria essencial ou muito importante para o aprendizado e $12,5 \%$ não responderam esta questão.

Após o preenchimento dos questionários pré-teste, os participantes foram convidados, aos pares, a realizar a atividade de exploração da plataforma Apuê. A exploração esteve concentrada na funcionalidade de Visualização e Controle de Experimentos. Os participantes tiveram a liberdade para explorar todos os elementos da interface e, quando possível, controlar remotamente o experimento. Sempre que necessário, os participantes podiam fazer perguntas aos pesquisadores. Apesar de não ter sido estipulada a duração das explorações, no geral, elas duraram cerca de 7 minutos. 
Após realizada a exploração, os pares de participantes foram convidados a responder o questionário pós-teste. Este questionário tinha o objetivo de contrastar a percepção após a atividade com a expectativa informada anteriormente e de analisar características pontuais da funcionalidade do Apuê (e.g., a qualidade do vídeo e das informações dos gráficos, a percepção de perda ou ganho do controle do experimento). Tal como no questionário préteste, a maioria das questões utilizou a escala Likert.

Pelo contraste com as respostas do questionário préteste é possível afirmar que a plataforma Apuê superou as expectativas da metade dos participantes, enquanto a outra metade afirmou que a plataforma correspondeu às suas expectativas (já bastante elevadas, de acordo com os resultados do pré-teste). Nenhum dos participantes considerou que a plataforma esteve abaixo de suas expectativas. Quando questionados mais especificamente quanto às expectativas em relação ao vídeo, em complemento ao que foi perguntado anteriormente no pré-teste, apenas $12,5 \%$ dos estudantes afirmaram que a visualização do vídeo ficou abaixo da expectativa. A qualidade do vídeo foi considerada boa por todos os estudantes, porém não ótima. A qualidade das informações do gráfico foi considerada boa ou ótima por $87,5 \%$ dos usuários, enquanto $12,5 \%$ a considerou ruim.

Um ponto delicado para a interatividade da plataforma era a percepção que o usuário teria quando recebesse ou perdesse o controle do experimento. Apenas 25\% dos estudantes perceberam imediatamente que tinham recebido o controle, enquanto que $62,5 \%$ perceberam somente após algum tempo e, ainda, 12,5\% perceberam apenas com a ajuda de um dos pesquisadores.

Com relação ao tempo de resposta dos comandos enviados ao experimento, $87,5 \%$ dos estudantes afirmaram que este ocorreu em tempo real ou com um ligeiro atraso, e $12,5 \%$ não responderam a esta questão. A tela do experimento foi considerada simples por $100 \%$ dos estudantes. Já, com relação à quantidade de informações presente na tela, metade dos participantes informou que a interface de usuário poderia ter mais informações, enquanto a outra metade afirmou que as informações, contidas na tela, eram suficientes.

Ainda no questionário pós-teste, os estudantes poderiam complementar suas respostas anteriores e fazer comentários referentes ao Apuê. A seguir alguns exemplos de comentários realizados: "Tive certa dificuldade no início para saber (identificar) o ponto no qual a F.C. [força centrípeta] seria igual ao peso inicial de sustentação. Mas depois que entendi certinho foi uma boa experiência, tanto no controle, quanto fora do mesmo"; "Dificuldade de perceber a mudança de quem controla o experimento. Seria interessante adotar uma solução utilizando cores para tornar mais chamativo".
Além das informações coletadas por meio dos questionários, os pesquisadores também fizeram anotações durante a exploração. Um dos fatos que chamaram a atenção dos pesquisadores foi que $25 \%$ dos participantes tentaram inserir dados nos campos de dados de saída, campos estes destinados apenas a mostrar os dados provindos dos sensores do experimento. $\mathrm{O}$ fato mais alarmante, constatado pelos pesquisadores, foi que $62,5 \%$ dos participantes tiveram dúvidas ou não perceberam a perda ou ganho do controle do experimento, assim, demonstrando uma falha no mecanismo de consciência desenvolvido para informar ao usuário sobre a perda/ganho do controle. Foi constatado, também, que $62,5 \%$ dos participantes não encontraram dificuldades significativas para utilizar os gráficos. Ainda, 75\% deles conseguiram efetuar mudanças na velocidade habilmente, ou seja, conseguiram compreender bem como funcionam os dados de entrada.

Por fim, a análise das gravações das telas dos participantes foram úteis para a identificação de dois fatos não constatados anteriormente Três dos participantes estavam usando o gráfico quando perderam ou receberam o controle da plataforma e não perceberam isto imediatamente (os dois recursos da interface estão em locais opostos da tela do experimento). A maioria dos participantes usou os dados de entrada habilmente, no entanto, alguns deles, ao usar novamente esse recurso se esqueceram de pressionar o botão "Enviar Dados", o que pode sugerir a necessidade de um indicador gráfico para os dados ainda não submetidos. Um segunda alternativa seria enviar os dados assim que o participante alterasse o valor do campo de entrada, no entanto, em algumas situações, o participante pode ter a intenção configurar um conjunto de dados de entrada antes de submetê-los ou, ainda, conferir os dados antes de enviá-los.

\subsection{Discussão}

A revisão de literatura adotou critérios de comparação das plataformas existentes, considerando o contexto brasileiro (por meio do questionário aplicados a alunos e professores do ensino médio e do superior). Nesta análise, a plataforma Apuê foi situada com os critérios considerados ideais. Após a conclusão da pesquisa é possível analisar quando tais critérios foram efetivamente atendidos e como o foram.

O primeiro critério, Aplicação Rica, foi alcançado pela conjunção de tecnologias para troca de mensagens assíncronas ( $2^{\circ}$ critério, sincronismo) entre cliente e servidor web (chamadas AJAX periódicas), streaming de vídeo (FFMPEG, Node.js e WebSocket) e gráficos dinâmicos (HighStock).

No decorrer do trabalho ocorreram inúmeras dificuldades e desafios que confrontaram sua viabilidade. Pro- 
vou-se um grande desafio técnico integrar todas as tecnologias necessárias para o funcionamento almejado da plataforma Apuê, pois diversas dessas ainda estão em estágio inicial de desenvolvimento, e.g., a tag "vídeo" do HTML5 ( $5^{\circ}$ critério, vídeo). Porém, apesar dos desafios, foi possível criar a plataforma mantendo o escopo da proposta inicial, i.e., uma solução extensível ( $6^{\circ}$ critério), de baixo custo, baseada inteiramente em padrões web $\left(7^{\circ}\right.$ critério) e tecnologias livres ( $3^{\circ}$ e $4^{\circ}$ critérios).

A extensibilidade é um dos pontos cruciais da plataforma Apuê. A plataforma provê recursos para que novos experimentos possam ser acoplados à plataforma, sem a necessidade de codificação na plataforma Web. Tanto a funcionalidade de Agendamento de Sessões quanto a de Visualização e Controle de Experimentos são automaticamente ajustadas às especificidades dos experimentos. Por exemplo, é possível adicionar quantos gráficos, dados de entrada e dados e saída forem necessários para manipulação e acompanhamento dos resultados dos experimentos. Para isso, basta o professor/administrador realizar as alterações no cadastro do experimento para que elas sejam automaticamente aplicadas às demais funcionalidades da plataforma.

Um diferencial relevante da plataforma Apuê é o fato de ela ter sido projetada para ser um módulo do AVA Sakai. Dessa maneira, ao instanciar a plataforma, a instituição de ensino tem à disposição não somente o Apuê, mas também diversos recursos relacionados à $\mathrm{EaD}$, tais como, repositório de arquivos, fórum, cadastro de cursos, grupos de participantes, entre outros.

Existem diversas possibilidades de extensões e melhorias para a plataforma. O estudo de caso revelou que há a necessidade de aprimorar o sistema de consciência sobre o ganho ou perda de controle do experimento, visando torná-lo mais perceptível; a representação mais apropriada dos dados de saída, de maneira que não sejam confundidos com elementos alteráveis; e a adição de maiores informações explicativas sobre o experimento, sob o ponto de vista da Física.

A escalabilidade de uma plataforma colaborativa e que envolve transmissão contínua de vídeo e outros dados é uma preocupação para a plataforma Apuê. O projeto da plataforma favorece a modularização como, por exemplo, a fácil separação dos servidores: web, de vídeo e de acoplamento de experimentos. No entanto, não foram realizadas medições empíricas para cenários de maiores volumes de acessos concorrentes.

As práticas laboratoriais contribuem para o aprendizado dos estudantes, no ensino das Ciências. Por esse motivo, a possibilidade de agregar a experiência dessas aulas em laboratório, com todos os benefícios previamente discutidos de um AVA, é potencialmente útil à comunidade científica e à sociedade. A descentralização geo- gráfica, redução de custos e maior alcance da educação são alguns dos benefícios provenientes dessa relação.

\section{Considerações finais}

Este artigo apresentou a concepção e avaliação da plataforma Apuê, uma plataforma para educação de ciências experimentais remotas de baixo custo e baseada em tecnologias livres. Esta plataforma provê recursos para a realização de práticas laboratoriais em cenários em que estudantes estejam localizados remotamente, tal como na EaD. Outra possibilidade de uso para este tipo de plataforma é na manipulação de experimentos que ofereçam riscos às pessoas ou que necessitem de um ambiente controlado para sua execução.

A pesquisa realizada para a concepção da plataforma Apuê traz contribuições relevantes em relação: (1) ao levantamento de requisitos realizado, que envolveu estudantes e professores do ensino médio e do superior, (2) a articulação das tecnologias emergentes, visando a redução de custos e maior compatibilidade com navegadores web e dispositivos, (3) a possibilidade de reuso da plataforma, por utilizar somente tecnologias livres, tanto de hardware quanto de software, e (4) a possibilidade de acoplamento de novos experimentos, por meio de interação com a interface gráfica da plataforma e sem a necessidade de conhecimentos em linguagens de programação.

Trabalhos futuros envolvem o acoplamento de novos experimentos à plataforma, a solução de problemas pontuais identificados pelo estudo de caso e a utilização da plataforma de maneira concorrente, por diversas instituições de ensino, visando avaliar a escalabilidade da plataforma e o uso conjunto do Apuê com os demais módulos do AVA Sakai.

Agradecimentos. Agradecemos aos alunos e professores que contribuíram para a modelagem e avaliação do Apuê, ao professor Rodrigo Ricetti (UTFPR) pela cessão do experimento de força centrípeta, ao Washington Máverick e ao PROGRAD/UTFPR, por apoiar a execução do projeto (Edital 21/2013).

\section{Referências}

[1] M. E. B. Almeida. Educação a distância na internet: abordagens e contribuições dos ambientes digitais de aprendizagem. Educação e Pesquisa (São Paulo). 29(2): 327-340, 2003.

[2] D. P. Ausubel, J. D. Novak, H. Hanesian. Educational Psychology: A Cognitive View. Holt, Rinehart and Winston, Inc., New York, 1978. 
[3] P. A. Berezuk, P. Inada. Avaliação dos laboratórios de ciências e biologia das escolas públicas e particulares de Maringá, Estado do Paraná. Acta Scientiarum. Human and Social Sciences. 32(2): 207-215, 2010.

[4] Brasil. Ensino por correspondência, SEPS, Brasília, 1980.

[5] V. S. Guimarães, I. N. de Oliveira, M. S. S. Lopes, C. R. S. de Oliveira, J. A. Oliveira, R. M. Reis. Um Ambiente para as Práticas Laboratoriais Remotas de Física: Estudo do Pêndulo Matemático. Revista Brasileira de Informática na Educação. 21(2): 78-91, 2013.

[6] B. Hanson, P. Culmer, J. Gallagher, K. Page, E. Read, A. Weightman, M. Levesley. ReLOAD: Real Laboratories Operated at a Distance. IEEE Transactions on Learning Technologies. 2(4): 331-341, 2009.

[7] R. Heineck, E. R. A. Valiati, C. T. W. Rosa. Software educativo no ensino de Física: análise quantitativa e qualitativa. Revista Iberoamericana de Educación. 42(6):1-12, 2007.

[8] INEP - Instituto Nacional de Estudos e Pesquisas Educacionais Anísio Teixeira. Resumo Técnico Censo da Educação Superior de 2011, 2011.

[9] K. Jarvi. RXTX: The Prescription for Transmission. http://users.frii.com/jarvi/rxtx/index.html, 4 de fev. de 2009.

[10] M. Jennings. Using The Sakai Maven Archetypes to Get Started Creating Tools. In JASIG (Java in Administration Special Interest Group) Sakai Conference, Atlanta, 2012.

[11] F. S. Junior, J. S. E. Germano, F. Almeida, M. C. Junior. Weblab e os experimentos de calorimetria. In XXXVII Congresso Brasileiro de Educação em Engenharia, 2008.

[12] Kogent Learning Solutions Inc. AJAX Black Book. Dreamtech Press, 2008.

[13] R. Likert. A Technique for the Measurement of Attitudes. Archives of Psychology, 140: 1-55, 1932.

[14] J. L. G. Llamas. El Estudio Empirico sobre el Rendimiento Academico en la Ensenanza a Distancia. ICE/UNEDE, Madri, 1986.

[15] C. J. P. Lucena, H. Fuks, R. Milidiu, L. Macedo, N. Santos, C. Laufer, M. B. Ribeiro, M. F. Fontoura, R. C. Noya, S. Crespo, V. Torres, L. Daflon, L. LUKOWIECKI (1998). AulaNet -- an environment for the development and mainte- nance of courses on the Web. In: Proceedings of the International Conference on Engineering in Education. Rio de Janeiro, RJ.

[16] G. L. Lucena, V. D. dos Santos, A. G. da Silva. Laboratório virtual como alternativa didática para auxiliar o ensino de química no ensino médio. Revista Brasileira de Informática na Educação. 21(2): 27-36, 2013.

[17] F. Marinelli, J. L. A. Pacca. Uma interpretação para dificuldades enfrentadas pelos estudantes em um laboratório didático de Física. Revista Brasileira de Ensino de Física. 28(4): 497-505, 2006.

[18] A. Niskier. Educação a distância: a tecnologia da esperança: políticas e estratégias para a implantação de um sistema nacional de educação aberta a distância, 2, Loyola, São Paulo, 2000.

[19] M. Nowotny. Interactive-matter/aJson - GitHub. https://github.com/interactive-matter/aJson, 03 de mar. 2014.

[20] C. R. Oliveira, I. Oliveira, H. Santos, A. Pereira. Um Ambiente para a Prática Remota de Aulas Laboratoriais de Física (determinação da viscosidade de líquidos). Revista Brasileira de Informática na Educação. 17(1): 43-57, 2009.

[21] Paraná, Secretaria de Estado da Educação. Superintendência da Educação. Educação a Distância. SEED-PR, 2010.

[22] O. Peters. Didática do Ensino a Distância. Editora Unisinos, São Leopoldo (RS), 2006.

[23] L. G. Santos. Desenvolvimentos em hardware e software para experimentos de física via Web. Trabalho de Conclusão de Curso. Universidade Estadual de Feira de Santana Departamento de Física, 2006.

[24] C. P. Souza, J. T. Costa Filho. Uma plataforma baseada na Web para ensino orientado a experimentos. In: International Conference on New Technologies in Science Education, 2001.

[25] L. Torre, J. Sánchez, S. Dormido. The Fis1@Bs Portal: A Network of Virtual and Remote Laboratories for Physics Education. In $14^{\text {th }}$ International Workshop on Multimedia in Physics Teaching and Learning, 2009. 